\title{
复合金属氧化物催化剂在光驱动合成过氧化氢中 的原位转化及其性能研究
}

\author{
韩丹丹, 刘健仪, 杨俊佼, 项项 * \\ 化工资源有效利用国家重点实验室, 北京化工大学, 北京 100029 \\ *通讯作者, E-mail: xiangxu@mail.buct.edu.cn
}

收稿日期: 2016-12-19; 接受日期: 2017-02-21; 网络版发表日期: 2017-03-30

国家重点基础研究发展计划(编号: 2014CB932104)、国家自然科学基金(编号: 21576016, U1507202, 21521005)和北京市自然科学基金(编 号: 2152022)资助项目

\begin{abstract}
摘要利用钛基复合金属氢氧化物作为前驱体, 通过焙烧转化为钛基复合金属氧化物, 作为光合成过氧化氢 的催化剂. 发现复合金属氧化物中的氧化锌晶相在合成过氧化氢反应中被原位溶蚀, 逐步转变为 $\mathrm{TiO}_{2}-\mathrm{ZnTiO}_{3}$ 相. 复合金属氧化物与原位转化得到的 $\mathrm{TiO}_{2}-\mathrm{ZnTiO}_{3}$ 都显示出比商品化的 $\mathrm{TiO}_{2}(\mathrm{P} 25: 80 \%$ 锐钛矿和 $20 \%$ 金红 石混合相)更好的光合成过氧化氢性能. 动力学分析表明, 过氧化氢的生成由其生成速率与分解速率共同 决定, $\mathrm{TiO}_{2}-\mathrm{ZnTiO}_{3}$ 作为催化剂时, 过氧化氢的生成速率常数 $\left(K_{\mathrm{f}}\right)$ 是 $3.581 \mu \mathrm{M} / \mathrm{min}$, 是复合金属氧化物作催化 剂时 $\left(K_{\mathrm{f}}=0.982 \mu \mathrm{M} / \mathrm{min}\right)$ 的 3.65 倍, 是 $\mathrm{P} 25\left(K_{\mathrm{f}}=0.852 \mu \mathrm{M} / \mathrm{min}\right)$ 的 4.20 倍. 而相应的分解速率常数 $\left(K_{\mathrm{d}}\right), \mathrm{TiO}_{2}-\mathrm{ZnTiO}_{3}$ $\left(K_{\mathrm{d}}=0.0472 \mathrm{~min}^{-1}\right)$ 是复合金属氧化物 $\left(K_{\mathrm{d}}=0.0119 \mathrm{~min}^{-1}\right)$ 的 3.97 倍, 是 $\mathrm{P} 25\left(K_{\mathrm{d}}=0.0374 \mathrm{~min}^{-1}\right)$ 的 1.26 倍. $\mathrm{TiO}_{2}-\mathrm{ZnTiO}_{3}$ 具有更大的生成速率常数和分解速率常数. 因此, 尽管 $\mathrm{TiO}_{2}-\mathrm{ZnTiO}_{3}$ 在反应初期催化生成了更多的过氧化氢, 但随着反应进行, $\mathrm{TiO}_{2}-\mathrm{ZnTiO}_{3}$ 催化合成的过氧化氢与复合金属氧化物达到几乎相同量, 这表明催化剂的原 位结构(物相)转变对反应动力学产生了影响, 但并不显著改变过氧化氢的最终产量. 抑制过氧化氢在催化 剂表面的分解是提高其产量的重要途径. 该工作加深了对钛基复合金属氧化物光催化合成过氧化氢的反应 动力学认识, 为钛基催化剂结构设计提供了一定指导.
\end{abstract}

关键词复合金属氧化物, 过氧化氢, 光合成, 反应动力学

\section{1 引言}

过氧化氢 $\left(\mathrm{H}_{2} \mathrm{O}_{2}\right)$ 被誉为“清洁”的化学品, 应用于 造纸、纺织、印染、电子、食品、环保和化学合成等 众多领域 ${ }^{[1]}$. 目前工业上生产 $\mathrm{H}_{2} \mathrm{O}_{2}$ 的主要方法是葱醌 法 $^{[2]}$, 具有装置易于大型化生产、产率较高等优点. 不 足之处是生产系统复杂、生产过程耗能较大, 当 $\mathrm{H}_{2} \mathrm{O}_{2}$
与有机物质同时存在时, 有爆炸和高毒性等危险. 因 此, Hutchings等 ${ }^{[3,4]}$ 提出了以 $\mathrm{Au} 、 \mathrm{Pd}$ 或AuPd双金属作 为催化剂, 通过 $\mathrm{H}_{2}$ 和 $\mathrm{O}_{2}$ 直接反应, 选择性生成 $\mathrm{H}_{2} \mathrm{O}_{2}$. 该 法基本不使用有机溶剂, 因此较葱醌法更绿色、环保. 但是, $\mathrm{H}_{2}$ 和 $\mathrm{O}_{2}$ 混合体系在很宽的浓度范围内具有爆炸 风险, 难以控制, 在合成时需精细调节两者的比例, 或 加入稀释剂(如 $\mathrm{N}_{2} 、 \mathrm{Ar}$ 气), 对反应产生影响, 导致 $\mathrm{H}_{2} \mathrm{O}_{2}$

引用格式: 韩丹丹, 刘健仪, 杨俊佼, 项顼. 复合金属氧化物催化剂在光驱动合成过氧化氢中的原位转化及其性能研究. 中国科学: 化学, 2017, 47: 465-473 Han D, Liu J, Yang J, Xiang X. In-situ conversion and catalytic properties of mixed-metal oxide catalysts for photosynthesis of hydrogen peroxide. Sci Sin Chim, 2017, 47: 465-473, doi: 10.1360/N032016-00232 
选择性不高, 产率较低.

为避免 $\mathrm{H}_{2}$ 和 $\mathrm{O}_{2}$ 直接接触, Fujishima等 ${ }^{[5]}$ 设计了以 $\mathrm{TiO}_{2}$ 为催化剂、质子氢 $\left(\mathrm{H}^{+}\right)$代替分子氢 $\left(\mathrm{H}_{2}\right)$ 光合成 $\mathrm{H}_{2} \mathrm{O}_{2}$, 但生成的 $\mathrm{H}_{2} \mathrm{O}_{2}$ 会在 $\mathrm{TiO}_{2}$ 表面快速分解, 降低 $\mathrm{H}_{2} \mathrm{O}_{2}$ 产率. Hirai等 ${ }^{[6]}$ 设计了 $\mathrm{TiO}_{2}$ 负载 $\mathrm{Au}-\mathrm{Ag}$ 双金属作为催化剂, 利 用贵金属将光生电子迅速转移出, 使 $\mathrm{H}_{2} \mathrm{O}_{2}$ 在贵金属表 面形成, 从而抑制 $\mathrm{H}_{2} \mathrm{O}_{2}$ 的分解, 提高 $\mathrm{H}_{2} \mathrm{O}_{2}$ 的产率. 然而, 探索替代贵金属的催化剂一直是一个挑战 ${ }^{[7]}$. Maurino 等 ${ }^{[8]}$ 提出了利用氟离子 $\left(\mathrm{F}^{-}\right)$改性 $\mathrm{TiO}_{2}$ 表面, 可以减少 $\mathrm{H}_{2} \mathrm{O}_{2}$ 的分解, 但氟化氢( $\mathrm{HF}$ ) 具有强腐蚀性, 操作风险 大. 因此, 寻找非贵金属催化剂、在温和条件下清洁 生产 $\mathrm{H}_{2} \mathrm{O}_{2}$ 具有重要意义.

以水和氧气为原料光合成 $\mathrm{H}_{2} \mathrm{O}_{2}$ 涉及水氧化和氧 还原反应, 设计具有双功能的催化剂是关键. 层状双 金属氢氧化物(LDHs) 由于独特的二维结构和灵活调 变的化学组成, 近年在水氧化催化反应中受到广泛关 注 $^{[0 \sim 15]}$. 以 $\mathrm{LDHs}$ 为前体, 经焙烧可拓扑转变为复合金 属氧化物(MMO), 许多含过渡金属的 $\mathrm{MMO}$ 是优异的 水氧化催化剂. 而通过前体法获得的 MMO具有特殊 的结构优势: 即金属离子在LDHs结构中有序排布, 拓 扑转变得到的MMO具有金属氧化物组分高分散特征, 有利于活性位充分暴露, 从而提高催化活性. 因此, 以 ZnTi-LDHs 为前体, 经焙烧得到ZnTi-MMO, 光驱动催 化合成 $\mathrm{H}_{2} \mathrm{O}_{2}$. 发现反应过程中 $\mathrm{ZnO}$ 晶相的原位溶蚀, 研 究了 $\mathrm{H}_{2} \mathrm{O}_{2}$ 生成和分解反应动力学, 探讨了 $\mathrm{MMO}$ 结构 转变对反应动力学的影响, 为 $\mathrm{H}_{2} \mathrm{O}_{2}$ 合成用 $\mathrm{Ti}$ 基催化剂 结构设计提供了指导.

\section{2 实验部分}

\section{1 试剂与仪器}

硝酸锌、异丙醇钛、脲均为分析纯试剂, 购于西 陇化工(北京)股份有限公司 (中国); 磷酸二氢钾、磷 酸氢二钾均为分析纯试剂, 购于国药集团(北京)化学 试剂有限公司(中国); 异丙醇、高氯酸、过氧化氢均 为分析纯试剂, 购于北京化工厂 (中国); 过氧化物酶为 生化试剂, 购于兰博利德(北京)商贸有限公司(中国); $N, N$-二乙基-1,4-苯二胺硫酸盐购于阿达玛斯(中国); 氧 气(99.995\%)购于海普气体(北京)有限公司(中国).

X射线衍射仪 (XRD, Shimadzu XRD-6000型, 日本. 测试条件: $\mathrm{Cu}$ 靶, $\mathrm{K} \alpha$ 射线源, 波长为 $1.5406 \AA$; 仪器 的测量误差为 $\pm 0.02^{\circ}$, 扫描角度范围 $3^{\circ} \sim 70^{\circ}$, 扫描速
率是 $10^{\circ} / \mathrm{min}$, 狭缝宽度是 $1^{\circ}$ ); 比表面-孔径分析 (BET, Micromeritics ASAP-2020型, 美国. 测试条件: 在液氮 温度 $\left(-196^{\circ} \mathrm{C}\right)$ 下测试材料的比表面积以及孔径分布); 透射电镜-能量散射谱(TEM-EDS, JEOL JEM-2010F型, 日本. 测试条件: 透射电镜配置OxFord X-Max ${ }^{\mathrm{N}} 80$-TLE 型能量散射配件, 通过面扫描(EDS mapping)表征出样 品中不同元素的分布); X射线光电子能谱仪 (XPS, Thermo VG ESCALAB MK II型, 美国. 测试条件: Al 阳极靶作为发射源, $\mathrm{Al} \mathrm{K \alpha}=1486.6 \mathrm{eV}$, 真空度是 $3 \times 10^{-9}$ $\mathrm{Pa}$, 光源的电压和电流分别为 $15 \mathrm{kV} \times 20 \mathrm{~mA}$, 使用 $\mathrm{C}$ 峰 $(284.8 \mathrm{eV})$ 进行校准); 紫外-可见吸收光谱仪(UV-Vis, UV-3000型, 岛津, 日本. 测试条件: 测试 $\mathrm{H}_{2} \mathrm{O}_{2}$ 浓度选 择的吸光波长 $551 \mathrm{~nm}$ ).

\section{2 锌钛复合金属氢氧化物的制备}

室温下准确称取 $8.922 \mathrm{~g} \mathrm{Zn}\left(\mathrm{NO}_{3}\right)_{2} \cdot 6 \mathrm{H}_{2} \mathrm{O} 、 2.842 \mathrm{~g}$ $\mathrm{Ti}\left[\mathrm{OCH}\left(\mathrm{CH}_{3}\right)_{2}\right]_{4} 、 6.005 \mathrm{~g}$ 脲于 $250 \mathrm{~mL}$ 烧杯中, 加入 $100 \mathrm{~mL}$ 去离子水, 超声、搅拌至混合形成均一溶液, 在 $20^{\circ} \mathrm{C}$ 下, 以 $40 \mathrm{~W}$ 的功率超声 $10 \mathrm{~min}$, 配制得到混合盐 溶液, 其中锌钠摩尔浓度比为 $3: 1$. 将混合液移至三口 烧瓶中在 $100^{\circ} \mathrm{C}$ 下加热搅拌回流 $48 \mathrm{~h}$, 冷却至室温, 抽 滤洗涤至 $\mathrm{pH}=7,60^{\circ} \mathrm{C}$ 干燥 $12 \mathrm{~h}$, 得到锌钛复合金属氢 氧化物(ZnTi-LDH).

\section{3 锌针复合金属氧化物的制备}

将上述复合金属氢氧化物(ZnTi-LDH)置于马弗炉 中, 在空气气氛中焙烧, 焙烧条件为: 以 $5{ }^{\circ} \mathrm{C} / \mathrm{min}$ 的升 温速率升至 $600^{\circ} \mathrm{C}$, 保温 $2 \mathrm{~h}$, 得到锌钛复合金属氧化物 (ZnTi-MMO).

\section{$2.4 \mathrm{TiO}_{2}-\mathrm{ZnTiO}_{3}$ 晶相的制备}

将 $1 \mathrm{~g} / \mathrm{L}$ 的ZnTi-MMO水相悬浮液置于玻璃容器中, 在磁力搅拌下以 $10 \mathrm{~mL} / \mathrm{min}$ 的速度滴加 $1 \mathrm{M}$ 的 $\mathrm{HClO}_{4}$ 至 溶液 $\mathrm{pH}=3.0$. 然后将悬浮液高速离心(5000 rpm, $2 \mathrm{~min}$ ), 用去离子水洗涤 3 次, 置于 $60^{\circ} \mathrm{C}$ 烘箱中干燥 $8 \mathrm{~h}$, 得到 $\mathrm{TiO}_{2}-\mathrm{ZnTiO}_{3}$ 晶相.

\section{5 光合成 $\mathrm{H}_{2} \mathrm{O}_{2}$ 浓度测定}

测试反应后溶液中 $\mathrm{H}_{2} \mathrm{O}_{2}$ 浓度参考文献 $[16]$ 的方 法. 将 $2.8756 \mathrm{~g} \mathrm{~K}_{2} \mathrm{HPO}_{4} \cdot 3 \mathrm{H}_{2} \mathrm{O}$ 和 $11.935 \mathrm{~g} \mathrm{KH}_{2} \mathrm{PO}_{4}$ 溶于 $200 \mathrm{~mL}$ 去离子水中, 配成 $1 \mathrm{M}$ 的磷酸缓冲液 $(\mathrm{pH}=7$ ). 将 $0.1 \mathrm{~g} \mathrm{~N}, \mathrm{~N}$-二乙基-1,4-苯二胺硫酸盐(DPD)溶于 $10 \mathrm{~mL}$ $0.05 \mathrm{M} \mathrm{H}_{2} \mathrm{SO}_{4}$ 溶液中; 将 $10 \mathrm{mg}$ 过氧化物酶(POD)溶于 
$10 \mathrm{~mL}$ 去离子水中配制成溶液. 将DPD和POD溶液放 入冰箱 $\left(3^{\circ} \mathrm{C}\right)$ 中保存待用. 移取 $0.8 \mathrm{~mL} 1 \mathrm{M}$ 的磷酸缓 冲液、 $2.24 \mathrm{~mL}$ 去离子水、 $100 \mu \mathrm{L}$ DPD溶液和 $100 \mu \mathrm{L}$ POD溶液混合到一起, 得到溶液 $\mathrm{A}$.

配制 $\mathrm{H}_{2} \mathrm{O}_{2}$ 标准溶液 $(0 、 10 、 50 、 100 \mu \mathrm{M})$, 用 $1000 \mu \mathrm{L}$ 的移液枪移取 $2 \mathrm{~mL}$ 配制好的 $\mathrm{H}_{2} \mathrm{O}_{2}$ 标准溶液 加入到溶液 $\mathrm{A}$ 中, 振荡 $30 \mathrm{~s}$ 后转移至石英比色㿼中, 用 UV-Vis测定不同浓度 $\mathrm{H}_{2} \mathrm{O}_{2}$ 标准溶液在 $551 \mathrm{~nm}$ 波长处 的吸光度值 $A_{C_{0}} 、 A_{C_{10}} 、 A_{C_{50}} 、 A_{C_{100}}$. 以 $\mathrm{H}_{2} \mathrm{O}_{2}$ 标准溶液的 浓度值为横坐标, 吸光度值为纵坐标作图, 并进行线 性拟合, 得到标准曲线.

向反应容器中加入 $28.5 \mathrm{~mL}$ 去离子水, 磁力摚拌 下滴加浓度为 $1 \mathrm{M}$ 的 $\mathrm{HClO}_{4}$ 至溶液 $\mathrm{pH}=3.0$, 再加入 30 $\mathrm{mg}$ 的催化剂和 $1.5 \mathrm{~mL}$ 异丙醇 (5 vol\%), 在无光照磁力 搅拌的条件下持续通入 $\mathrm{O}_{2} 30 \mathrm{~min}$, 使反应溶液中的溶 解氧达到饱和状态, 然后使用氙灯水平照射反应器反 应 $3.5 \mathrm{~h}$, 在光催化反应的前 $30 \mathrm{~min}$ 每隔 $10 \mathrm{~min}$ 定时取 样, 然后每隔 $30 \mathrm{~min}$ 定时取样. 用移液枪从烧杯中取 出 $2 \mathrm{~mL}$ 反应液加入至一次性注射器中, 通过孔径为 $0.45 \mu \mathrm{m}$ 的滤膜滤去固体催化剂粉末, 将得到的澄清溶 液转加入至溶液 $\mathrm{A}$ 中, 振荡 $30 \mathrm{~s}$ 后转移至石英比色血 中, 用UV-Vis测定各个时间所取样品在 $551 \mathrm{~nm}$ 波长处 的吸光度值 $A_{t}$. 由标准曲线求出吸光度值为 $A_{t}$ 时所对 应的 $\mathrm{H}_{2} \mathrm{O}_{2}$ 浓度 $C_{t}$.

\section{$2.6 \mathrm{TiO}_{2}-\mathrm{ZnTiO}_{3}$ 重复使用}

将光反应后的溶液高速离心(5000 rpm, $2 \mathrm{~min}$ ), 用 去离子水洗涤 3 次, 将得到的离心固体沉淀置于 $60^{\circ} \mathrm{C}$ 烘箱中干燥 $8 \mathrm{~h}$, 然后在相同的反应条件下测试光合成 $\mathrm{H}_{2} \mathrm{O}_{2}$ 性能.

\section{$2.7 \mathrm{H}_{2} \mathrm{O}_{2}$ 在催化剂上的分解测试}

向反应容器中加入 $28.5 \mathrm{~mL}$ 去离子水, 磁力搅拌下 滴加浓度为 $1 \mathrm{M}$ 的 $\mathrm{HClO}_{4}$ 至溶液 $\mathrm{pH}=3.0$, 再加入 $30 \mathrm{mg}$ 的催化剂和 $1.5 \mathrm{~mL}$ 异丙醇 (5 vol\%), 在无光照、磁力摚 拌的条件下持续通入 $\mathrm{O}_{2} 30 \mathrm{~min}$ 使反应溶液中溶解氧 达到饱和状态, 然后加入 $30 \% \mathrm{H}_{2} \mathrm{O}_{2}\left(C_{0}=5 \mathrm{mM}\right)$, 使用波 长 $>320 \mathrm{~nm}$, 光照强度为 $100 \mathrm{~mW} / \mathrm{cm}^{2}$ 的氙灯光源照射 反应器 $90 \mathrm{~min}$, 每隔 $10 \mathrm{~min}$ 取样测定 $\mathrm{H}_{2} \mathrm{O}_{2}$ 浓度.

\section{3 结果与讨论}

\subsection{XRD分析}

分析ZnTi-LDHs前体的XRD图谱(图1(a)), 得出所 有衍射峰与LDHs的特征峰吻合, 分别与(003)、(006)、 (012)、(101)、(009)、(107)、(108)、(113) 晶面相对 应, 没有出现其他衍射峰, 证明产物是LDHs晶相. 经 拓扑转变得到的ZnTi-MMO的图谱(图1(b)) 能够指标 化到 3 个晶相, 其中 $2 \theta$ 为 $25.28^{\circ}$ 处的衍射峰能指标化到 $\mathrm{TiO}_{2}(\mathrm{PDF} 21-1272)$ 的(101)晶面. $2 \theta^{\circ}$ 为 $31.77^{\circ} 、 34.42^{\circ}$ 、 $36.25^{\circ} 、 47.54^{\circ} 、 56.60^{\circ} 、 62.86^{\circ} 、 66.38^{\circ} 、 67.96^{\circ}$ 、 $69.10^{\circ}$ 处衍射峰对应于 $\mathrm{ZnO}$ (PDF 36-1451) 的 (100)、 (002)、(101)、(102)、(110)、(103)、(200)、(112)、 (201) 晶面. 对应于 $\mathrm{ZnTiO}_{3}$ 的特征峰强度较弱, 可能是 由于其含量相对较少, 且高度分散. $\mathrm{TiO}_{2}-\mathrm{ZnTiO}_{3}$ 的图谱 (图1(c))能指标化到两个晶相, 在 $2 \theta^{\circ}$ 为 $25.28^{\circ} 、 48.05^{\circ}$ 、 $53.89^{\circ}$ 处的衍射峰分别指标化到 $\mathrm{TiO}_{2}(\mathrm{PDF} 21-1272)$ 的(101)、(200)、(105) 晶面. 在 $2 \theta$ 为 $30.03^{\circ} 、 35.38^{\circ}$ 、 $42.99^{\circ} 、 56.86^{\circ} 、 62.44^{\circ}$ 的衍射峰对应于 $\mathrm{ZnTiO}_{3}(\mathrm{PDF}$ $36-0190)$ 的(220)、(311)、(400)、(511)、(440)晶面, 没 有出现氧化锌的衍射峰, 证明氧化锌可在反应的酸性
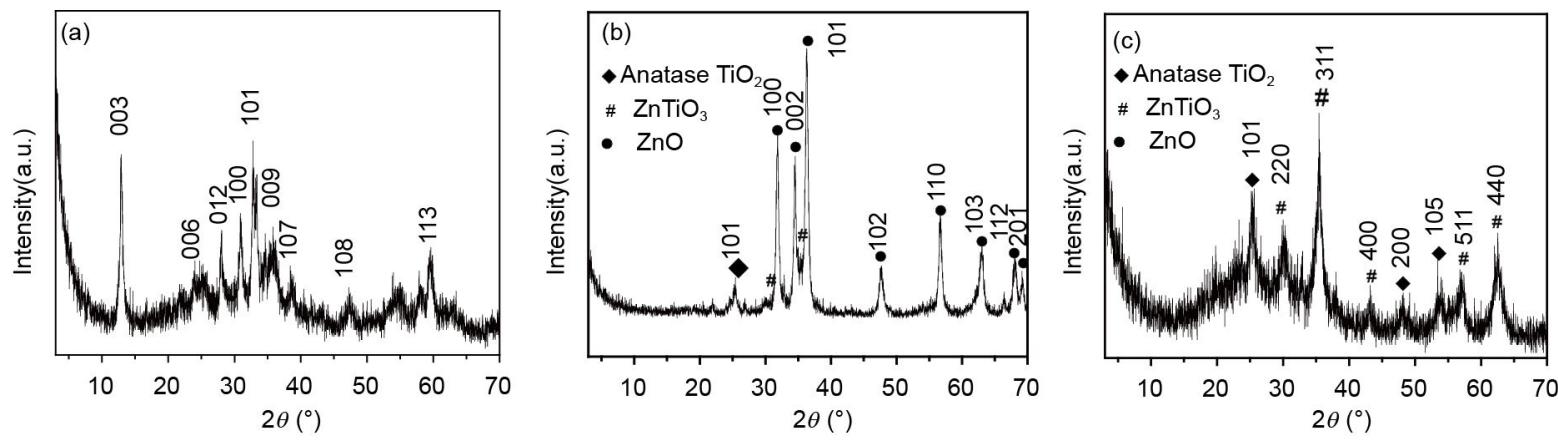

图 1 ZnTi-LDHs (a), ZnTi-MMO (b), $\mathrm{TiO}_{2}-\mathrm{ZnTiO}_{3}$ (c)的XRD图谱 
条件下被溶蚀, 得到 $\mathrm{TiO}_{2}-\mathrm{ZnTiO}_{3}$ 相 ${ }^{[17,18]}$.

\section{$3.2 \mathrm{UV}$-Vis分析}

利用UV-Vis研究催化剂的光响应行为. 以 $F(R)$ 为纵 坐标, 波长为横坐标得到样品的漫反射光谱(图2), 其中 $R$ 是漫反射率, $F(R)$ 由Kubelka-Munk方程 $F(R)=(1-R)^{2} / 2 R$ 得出. 所研究的钛基催化剂与氧化锌样品的光响应波长 在紫外光区, $\mathrm{ZnO}$ 具有更强的光响应. 相比 $\mathrm{TiO}_{2}-\mathrm{ZnTiO}_{3}$, ZnTi-MMO有较强的光响应可归因于ZnTi-MMO中ZnO 晶相的贡献. UV-Vis结果表明, 钛基催化剂(ZnTi$\mathrm{MMO} 、 \mathrm{TiO}_{2}-\mathrm{ZnTiO}_{3} 、 \mathrm{P} 25$ ) 在可见光区 (波长 $>400 \mathrm{~nm}$ ) 均无光响应, 这是由于 $\mathrm{TiO}_{2}$ (带隙 $3.2 \mathrm{eV}$ ) 和 $\mathrm{ZnTiO}_{3}$ (带 隙 $3.65 \mathrm{eV}$ ) 具有较宽的带隙. 改善目前钛基催化剂的可 见光响应能力将是提高其对光利用效率的重要途径.

\subsection{XPS 分析}

XPS 分析能给出催化剂中各元素的价态与结 合状态. ZnTi-MMO 和 $\mathrm{TiO}_{2}-\mathrm{ZnTiO}_{3}$ 的 XPS O1s 核能 级谱 (图 3(a)) 可拟合为 3 个峰, 其中结合能在 530.0 和531.9 eV处的峰归于晶格氧 $\left(\mathrm{O}^{2-}\right)$ 和表面吸附的 $\mathrm{H}_{2} \mathrm{O}$ 物种. 两个样品的晶格氧 $\left(\mathrm{O}^{2-}\right)$ 结合能没有明显差异, 表明溶蚀氧化锌后得到的 $\mathrm{TiO}_{2}-\mathrm{ZnTiO}_{3}$ 中的晶格氧物 种不发生改变. 在 $530.8 \mathrm{eV}$ 处的峰归于羟基物种 $\left(\mathrm{OH}^{-}\right)$. 进一步比较了各样品的 Zn2p核能级(图3(b)), Zn2p核 能级谱显示两个峰, 与参考样品 $\mathrm{ZnO}$ 相比, ZnTi-MMO 的 $Z n 2 p$ 结合能位置 $(1021.2$ 和 $1044.5 \mathrm{eV})$ 无明显移动, 这是由于 ZnTi-MMO中含大量 $Z n O$ 相, $Z n 2 p$ 结合能由 $\mathrm{ZnO}$ 贡献 ${ }^{[19]}$. 而 $\mathrm{TiO}_{2}-\mathrm{ZnTiO}_{3} \mathrm{Zn} 2 \mathrm{p}$ 核能级的结合能出现 在 1022.4 和 $1045.5 \mathrm{eV}$ (分别向高结合能方向移动了 1.2 和 $1.0 \mathrm{eV}$ ), 这是由于 $\mathrm{Zn}$ 物种来自 $\mathrm{ZnTiO}_{3}$ 相, 说明溶蚀 掉 $\mathrm{ZnO}$ 后, 得到了 $\mathrm{TiO}_{2}-\mathrm{ZnTiO}_{3}$ 相.

\subsection{BET 与孔结构分析}

对比了 $\mathrm{ZnTi}-\mathrm{MMO}$ 和 $\mathrm{TiO}_{2}-\mathrm{ZnTiO}_{3}$ 两个催化剂样品 的比表面积 $(S)$ 、孔体积 $\left(V_{\mathrm{p}}\right)$ 、孔尺寸(pore size) (表1). 分析结果得出, $\mathrm{TiO}_{2}-\mathrm{ZnTiO}_{3}$ 的比表面积 $\left(49.1 \mathrm{~m}^{2} / \mathrm{g}\right)$ 比 $\mathrm{ZnTi}-\mathrm{MMO}\left(40.7 \mathrm{~m}^{2} / \mathrm{g}\right)$ 略有增大, 表明溶蚀掉 $\mathrm{ZnO}$ 相, 对 $\mathrm{TiO}_{2}-\mathrm{ZnTiO}_{3}$ 的比表面积影响不大. $\mathrm{TiO}_{2}-\mathrm{ZnTiO}_{3}$ 与 $Z \mathrm{nTi}-\mathrm{MMO}$ 的孔体积分别为 0.20 和 $0.18 \mathrm{~cm}^{3} / \mathrm{g}$, 无明显 差别. 而 $\mathrm{TiO}_{2}-\mathrm{ZnTiO}_{3}$ 的孔尺寸 $(16 \mathrm{~nm})$ 相比 ZnTi-MMO $(11.6 \mathrm{~nm})$ 有一定增大, 这是由于 $\mathrm{ZnTi}-\mathrm{MMO}$ 中的 $\mathrm{ZnO}$ 相 被溶蚀后, 孔隙增加, 因此孔尺寸增大. 更大的孔尺寸 有利于反应物种、生成物种在催化剂表面的扩散、
传质, 提高反应速率.

\subsection{TEM-EDS 分析}

利用TEM-EDS对催化剂形貌和元素组成进行表

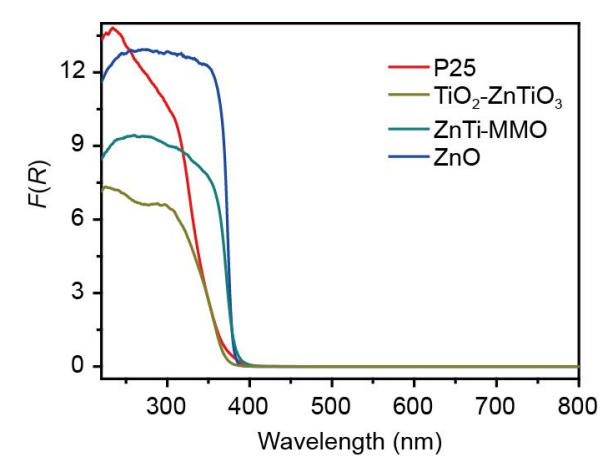

图 $2 \mathrm{TiO}_{2}-\mathrm{ZnTiO}_{3} 、 \mathrm{ZnTi}-\mathrm{MMO} 、 \mathrm{P} 25 、 \mathrm{ZnO}$ 样品的紫外-可 见漫反射光谱(网络版彩图)
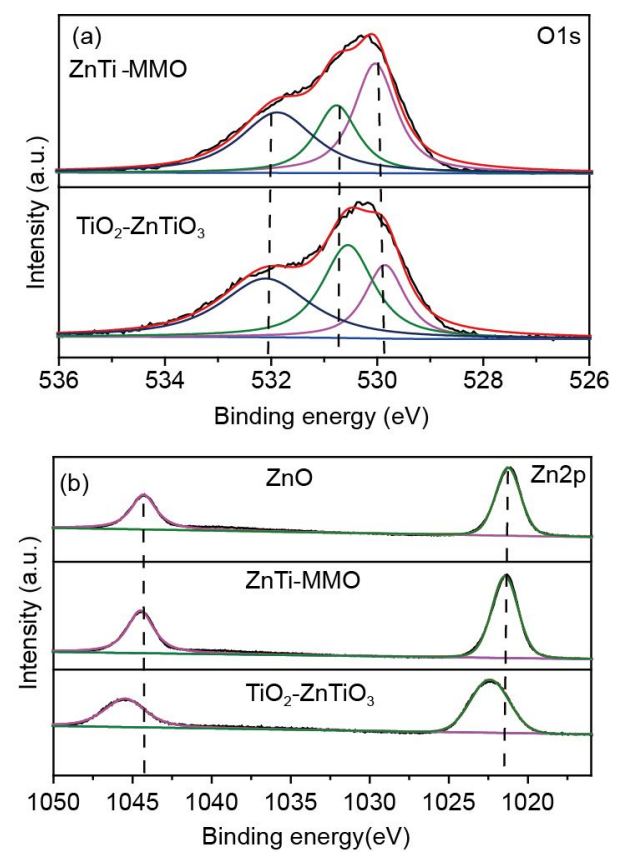

图 3 (a) $\mathrm{ZnTi}-\mathrm{MMO}$ 和 $\mathrm{TiO}_{2}-\mathrm{ZnTiO}_{3}$ 的XPS O1s核能级谱; (b) $\mathrm{ZnO} 、 \mathrm{ZnTi}-\mathrm{MMO}$ 和 $\mathrm{TiO}_{2}-\mathrm{ZnTiO}_{3}$ 的XPS Zn2p核能级谱(网络 版彩图)

表 $1 \mathrm{ZnTi-MMO}$ 和 $\mathrm{TiO}_{2}-\mathrm{ZnTiO}_{3}$ 的比表面积、孔体积、孔 尺寸比较

\begin{tabular}{cccc}
\hline Sample & $S\left(\mathrm{~m}^{2} / \mathrm{g}\right)$ & $V_{\mathrm{p}}\left(\mathrm{cm}^{3} / \mathrm{g}\right)$ & pore size $(\mathrm{nm})$ \\
\hline $\mathrm{ZnTi}-\mathrm{MMO}$ & 40.7 & 0.18 & 11.6 \\
$\mathrm{TiO}_{2}-\mathrm{ZnTiO}_{3}$ & 49.1 & 0.20 & 16.0 \\
\hline
\end{tabular}


征. 从 TEM 观察, ZnTi-MMO 中的颗粒尺寸约 20 $30 \mathrm{~nm}$, 分散较好, 无明显团聚(图4(a)). HRTEM观察发 现, 0.260 和 $0.162 \mathrm{~nm}$ 面间距的晶格条纹, 分别对应于 $\mathrm{TiO}_{2}$ 的(101)和 $\mathrm{ZnO}$ 的(102)晶面. 未观察到 $\mathrm{ZnTiO}_{3}$ 相的 晶格条纹, 这可能是由于 $Z n T i-M M O$ 中主要是 $Z n O$ 和 $\mathrm{TiO}_{2}$ 两种晶相. 这与XRD图谱的结果一致. 溶蚀氧化锌 后的 $\mathrm{TiO}_{2}-\mathrm{ZnTiO}_{3}$ 相观察到 $\mathrm{TiO}_{2}$ 和 $\mathrm{ZnTiO}_{3}$ 的相界面, 面 间距 0.260 和 $0.382 \mathrm{~nm}$ 分别对应于 $\mathrm{TiO}_{2}$ 的(101)和 $\mathrm{ZnTiO}_{3}$ 的(440)晶面(图4(b)).

与前述XRD图谱一致. 进一步对样品中的 Ti、 $\mathrm{Zn} 、 \mathrm{O}$ 元素进行了EDS mapping分析. 3种元素在ZnTi$\mathrm{MMO}$ 和 $\mathrm{TiO}_{2}-\mathrm{ZnTiO}_{3}$ 中都是均匀分布, 表明 $\mathrm{ZnO} 、 \mathrm{TiO}_{2}$ 和 $\mathrm{ZnTiO}_{3}$ 物相在样品中高度分散, 有利于催化活性物 相充分暴露, 与反应物更好地接触, 体现了LDHs前体 法制备MMO的优势.

\section{6 催化性能测试}

\subsection{1 光驱动合成 $\mathrm{H}_{2} \mathrm{O}_{2}$}

对合成 $\mathrm{H}_{2} \mathrm{O}_{2}$ 的实验条件进行逐一探究发现, 分子 氧 $\left(\mathrm{O}_{2}\right)$ 、催化剂和光照是必需条件, 三者缺一不可. 为比较钛基催化剂的性能, 在相同的实验条件下进 行合成 $\mathrm{H}_{2} \mathrm{O}_{2}$ 测试. 实验条件为: $1 \mathrm{~g} / \mathrm{L}$ 催化剂, 用浓度 $1 \mathrm{M}$ 的 $\mathrm{HClO}_{4}$ 调节反应水溶液的 $\mathrm{pH}$ 至 3.0 , 加入 $5 \mathrm{vol} \%$ 的异丙醇, 光照前向反应溶液中持续通入 $\mathrm{O}_{2} 30 \mathrm{~min}$ 至溶解氧达到饱和, 氙灯光源, 波长 $>320 \mathrm{~nm}$, 光照强
度 $100 \mathrm{~mW} / \mathrm{cm}^{2}$. 在催化剂 $\mathrm{TiO}_{2}-\mathrm{ZnTiO}_{3} 、 \mathrm{ZnTi}-\mathrm{MMO}$ 和 $\mathrm{P} 25$ 存在下光合成 $\mathrm{H}_{2} \mathrm{O}_{2}$ 的浓度-时间曲线, 如图 5(a) 所 示. 分析发现, 在 $\mathrm{TiO}_{2}-\mathrm{ZnTiO}_{3}$ 和 ZnTi-MMO作为催化 剂时, $\mathrm{H}_{2} \mathrm{O}_{2}$ 的最终产量(反应 $210 \mathrm{~min}$ )和生成速率均大 于 $\mathrm{P} 25$ 作为催化剂时. 对 $\mathrm{H}_{2} \mathrm{O}_{2}$ 的浓度-时间曲线进行动 力学拟合, 采用方程: $\left[\mathrm{H}_{2} \mathrm{O}_{2}\right]=\left(K_{\mathrm{f}} / k_{\mathrm{d}}\right)\left\{1-\exp \left(-K_{\mathrm{d}} t\right)\right\}^{[7]}$. 其 中 $K_{\mathrm{f}}$ 是 $\mathrm{H}_{2} \mathrm{O}_{2}$ 的生成速率常数, $K_{\mathrm{d}}$ 是 $\mathrm{H}_{2} \mathrm{O}_{2}$ 的分解速率常 数(图 5(b)). $\mathrm{H}_{2} \mathrm{O}_{2}$ 的生成与分解是竞争过程. 由于在反 应过程中 $\mathrm{O}_{2}$ 是持续不断地通入 (保持过量), 所以 $\mathrm{H}_{2} \mathrm{O}_{2}$ 的生成反应可以看作零级反应, 而 $\mathrm{H}_{2} \mathrm{O}_{2}$ 在催化剂表面 的分解反应可近似为一级反应. 分析动力学拟合结 果发现(表2), 在 $\mathrm{TiO}_{2}-\mathrm{ZnTiO}_{3}$ 催化剂上 $\mathrm{H}_{2} \mathrm{O}_{2}$ 的 $K_{\mathrm{f}}(3.581)$ 是ZnTi-MMO $\left(K_{\mathrm{f}}=0.982\right)$ 的 3.65 倍, 是 $\mathrm{P} 25\left(K_{\mathrm{f}}=0.852\right)$ 的 4.20 倍; 相应的 $\mathrm{H}_{2} \mathrm{O}_{2}$ 分解速率 $\mathrm{TiO}_{2}-\mathrm{ZnTiO}_{3}\left(K_{\mathrm{d}}=0.0472\right)$ 是ZnTi-MMO $\left(K_{\mathrm{d}}=0.0119\right)$ 的3.97倍, 是P25 $\left(K_{\mathrm{d}}=0.0374\right)$ 的 1.26 倍. $\mathrm{TiO}_{2}-\mathrm{ZnTiO}_{3}$ 作为光催化剂时, 尽管 $\mathrm{H}_{2} \mathrm{O}_{2}$ 的 生成速率快, 但其竞争反应一一分解速率也快, 这是 导致 $\mathrm{H}_{2} \mathrm{O}_{2}$ 在 $\mathrm{TiO}_{2}-\mathrm{ZnTiO}_{3}$ 和 $\mathrm{ZnTi}-\mathrm{MMO}$ 存在下, 反应结 束时最终产量几乎相同的原因. 因此, 要提高 $\mathrm{H}_{2} \mathrm{O}_{2}$ 的 产量, 抑制其在催化剂表面的分解是关键. 由于目前 的催化剂仅吸收紫外波段的光, 对光的利用有限, 会 影响催化合成 $\mathrm{H}_{2} \mathrm{O}_{2}$ 的效率. 所以调控催化剂的可见光 吸收能力也是增强光合成 $\mathrm{H}_{2} \mathrm{O}_{2}$ 性能的一个途径. 由于 反应是在 $\mathrm{pH}=3.0$ 的水溶液中进行, $\mathrm{ZnO}$ 相在这种条件 下会逐渐溶蚀. 在ZnTi-MMO存在时, $\mathrm{H}_{2} \mathrm{O}_{2}$ 的初始生 成速率(前 $30 \mathrm{~min}$ 反应) 低于 $\mathrm{TiO}_{2}-\mathrm{ZnTiO}_{3}$ 作为催化剂时
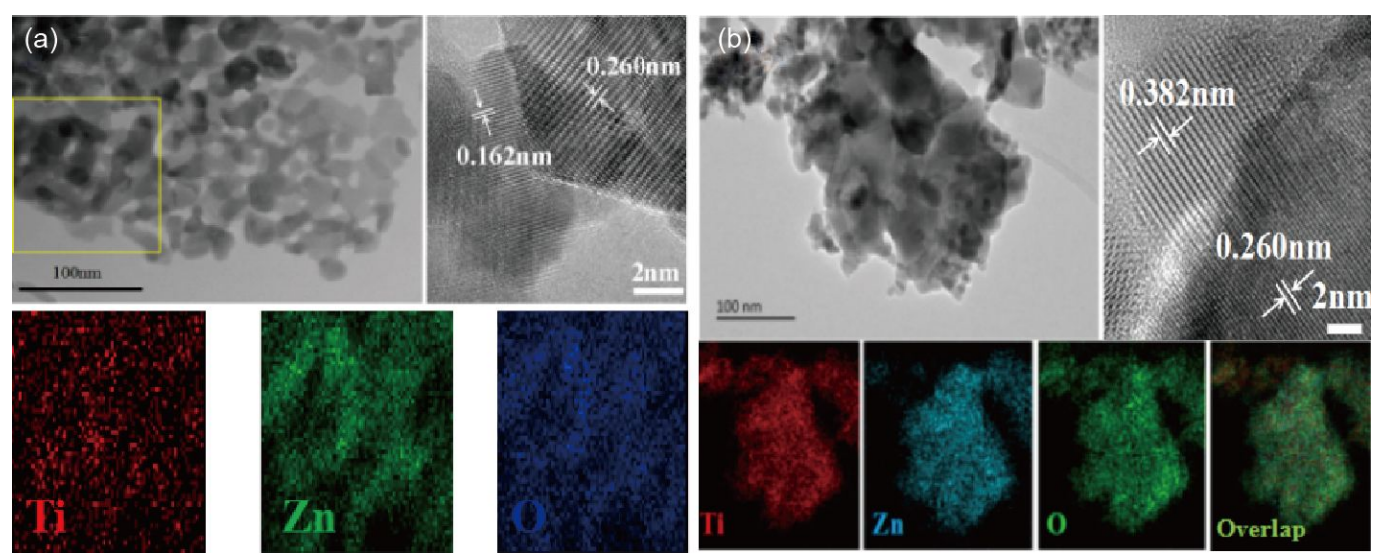

图 4 (a) ZnTi-MMO的TEM图, 和相应的 Ti、 $\mathrm{Zn} 、 \mathrm{O}$ 的元素EDS mapping 图; (b) $\mathrm{TiO}_{2}-\mathrm{ZnTiO}_{3}$ 的 TEM图, 和相应的 Ti、 $\mathrm{Zn} 、 \mathrm{O}$ 的元素EDS mapping图及 Ti、 Zn、O元素分布叠加图(Overlap) (网络版彩图) 

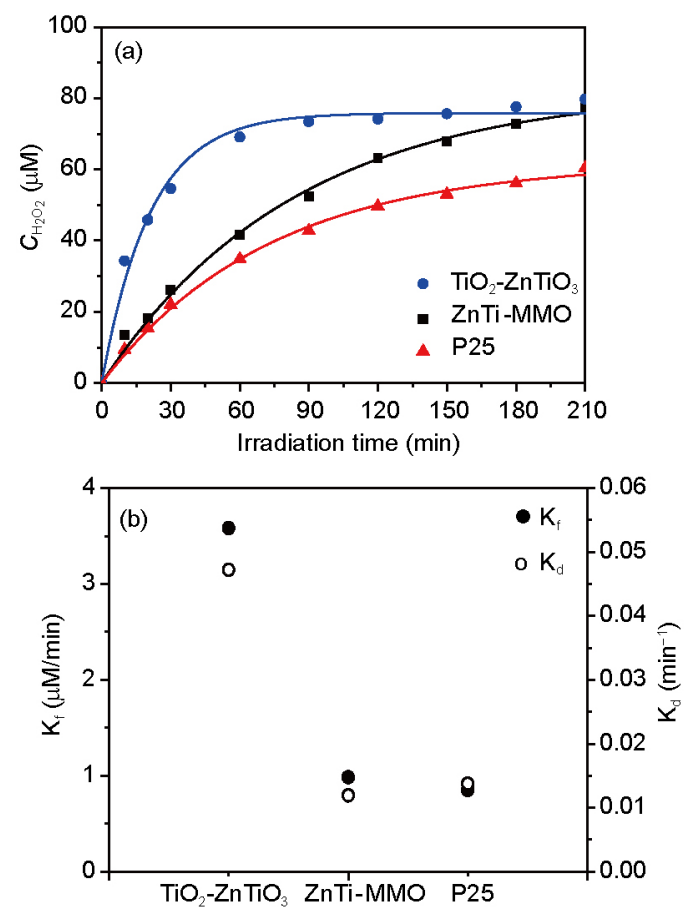

图 5 (a) 在催化剂存在下, 光驱动合成 $\mathrm{H}_{2} \mathrm{O}_{2}$ 的浓度-时间曲 线; (b) $\mathrm{H}_{2} \mathrm{O}_{2}$ 的生成速率常数 $\left(K_{\mathrm{f}}\right)$ 和分解速率常数 $\left(K_{\mathrm{d}}\right)$. 反应 条件为: $1 \mathrm{~g} / \mathrm{L}$ 的光催化剂, $5 \mathrm{vol} \%$ 的异丙醇, $\mathrm{pH}=3.0$, 氧平衡 状态, 氙灯光源, 波长 $>320 \mathrm{~nm}$, 光照强度为 $100 \mathrm{~mW} / \mathrm{cm}^{2}$ (网 络版彩图)

表 $2 \mathrm{H}_{2} \mathrm{O}_{2}$ 在催化剂 $\mathrm{TiO}_{2}-\mathrm{ZnTiO}_{3} 、 \mathrm{ZnTi}-\mathrm{MMO} 、 \mathrm{P} 25$ 存在 下的生成速率常数 $\left(K_{\mathrm{f}}\right)$ 和分解速率常数 $\left(K_{\mathrm{d}}\right)$

\begin{tabular}{ccc}
\hline Sample & $K_{\mathrm{f}}(\mu \mathrm{M} / \mathrm{min})$ & $K_{\mathrm{d}}\left(\mathrm{min}^{-1}\right)$ \\
\hline $\mathrm{TiO}_{2}-\mathrm{ZnTiO}_{3}$ & 3.5814 & 0.0472 \\
$\mathrm{ZnTi}-\mathrm{MMO}$ & 0.9851 & 0.01192 \\
$\mathrm{P} 25$ & 0.8509 & 0.01374 \\
\hline
\end{tabular}

(图5(a)). 尽管 $\mathrm{H}_{2} \mathrm{O}_{2}$ 在 $\mathrm{TiO}_{2}-\mathrm{ZnTiO}_{3}$ 上有更大的初始生 成速率, 但反应到 $90 \mathrm{~min}$ 时, $\mathrm{H}_{2} \mathrm{O}_{2}$ 的平衡浓度不再增大, 表明此时 $\mathrm{H}_{2} \mathrm{O}_{2}$ 的生成与分解达到反应动态平衡, 即使 延长反应时间, $\mathrm{H}_{2} \mathrm{O}_{2}$ 的浓度也不会增加. 在ZnTi-MMO 上 $\mathrm{H}_{2} \mathrm{O}_{2}$ 达到平衡浓度需要更长时间, 反应到 $210 \mathrm{~min}$ 时, $\mathrm{H}_{2} \mathrm{O}_{2}$ 的平衡浓度与在 $\mathrm{TiO}_{2}-\mathrm{ZnTiO}_{3}$ 上的几乎一样, 说明 有 $\mathrm{ZnO}$ 相存在时, $\mathrm{H}_{2} \mathrm{O}_{2}$ 的生成速率和分解速率较小, 随 着 $\mathrm{ZnO}$ 的原位溶蚀, $\mathrm{H}_{2} \mathrm{O}_{2}$ 的生成速率和分解速率增大. $\mathrm{ZnO}$ 相被完全溶蚀后, 即在 $\mathrm{TiO}_{2}-\mathrm{ZnTiO}_{3}$ 上 $\mathrm{H}_{2} \mathrm{O}_{2}$ 有更大 的生成速率与分解速率. 这初步证明对 $\mathrm{H}_{2} \mathrm{O}_{2}$ 生成起主 要贡献的是 $\mathrm{TiO}_{2}$ 和 $\mathrm{ZnTiO}_{3}$ 物相, 而形成 $\mathrm{TiO}_{2}-\mathrm{ZnTiO}_{3}$ 异
质结构能够提高光合成 $\mathrm{H}_{2} \mathrm{O}_{2}$ 的性能.

对比了以单位摩尔钛计算的 $\mathrm{H}_{2} \mathrm{O}_{2}$ 在催化剂上的生 成量(图6), 在反应到 $210 \mathrm{~min}$ 时, 在 $\mathrm{ZnTi}-\mathrm{MMO}$ 上 $\mathrm{H}_{2} \mathrm{O}_{2}$ 生成量达到 $782 \mathrm{mM}$, 是 $\mathrm{P} 25$ 的 4.86 倍. 在 $\mathrm{TiO}_{2}-\mathrm{ZnTiO}_{3}$ 上 $\mathrm{H}_{2} \mathrm{O}_{2}$ 生成量达到 $585 \mathrm{mM}$, 是P25的3.76倍. 这可能是由 于 $\mathrm{H}_{2} \mathrm{O}_{2}$ 在 $\mathrm{P} 25$ 上发生强烈分解反应, 显著降低 $\mathrm{H}_{2} \mathrm{O}_{2}$ 的 生成量 ${ }^{[7,8]}$. 而形成 $\mathrm{ZnTi}-\mathrm{MMO}$ 和 $\mathrm{TiO}_{2}-\mathrm{ZnTiO}_{3}$ 可以提高 Ti的利用率, 是活性组分有效利用的一条途径.

\subsection{2 催化剂循环使用性能}

催化剂的稳定性和循环使用性是衡量催化剂性 能的一个重要指标. 考察了 $\mathrm{TiO}_{2}-\mathrm{ZnTiO}_{3}$ 的重复使用性 能. 对光合成 $\mathrm{H}_{2} \mathrm{O}_{2}$ 反应后 $(210 \mathrm{~min})$ 的 $\mathrm{TiO}_{2}-\mathrm{ZnTiO}_{3}$ 进行 回收后再使用. 经过4次重复使用, 催化合成 $\mathrm{H}_{2} \mathrm{O}_{2}$ 的性 能没有发生明显下降(图7), 表明 $\mathrm{TiO}_{2}-\mathrm{ZnTiO}_{3}$ 具有很好 的稳定性和循环使用性.

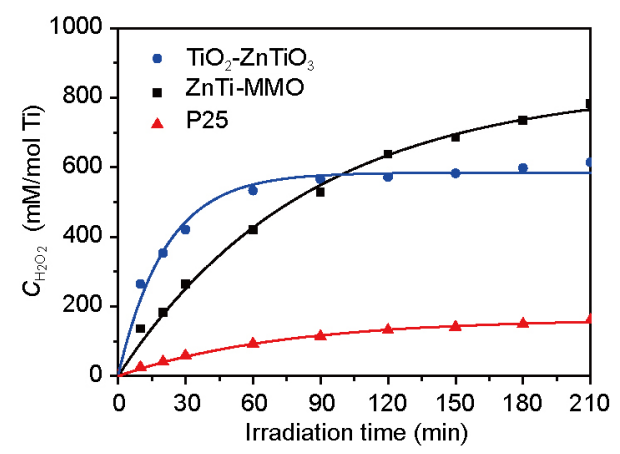

图 6 催化剂光驱动合成 $\mathrm{H}_{2} \mathrm{O}_{2}$ 性能曲线(以单位摩尔钛计). 反应条件为: $1 \mathrm{~g} / \mathrm{L}$ 的光催化剂, $5 \mathrm{vol} \%$ 的乙醇, $\mathrm{pH}=3.0$, 氧平 衡状态, 氙灯光源, 波长 $>320 \mathrm{~nm}$, 光照强度为 $100 \mathrm{~mW} / \mathrm{cm}^{2}$ (网 络版彩图)

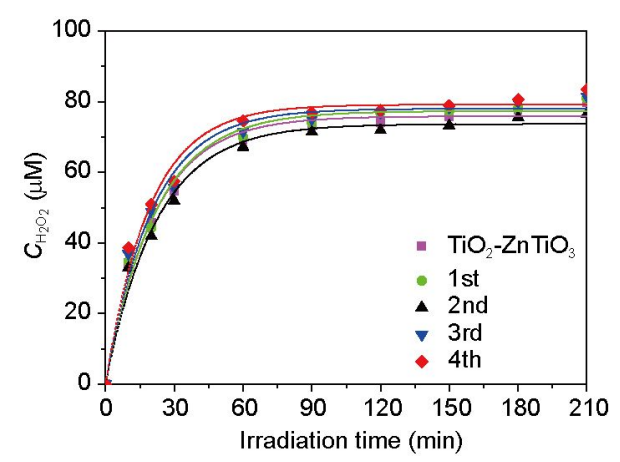

图 $7 \mathrm{TiO}_{2}-\mathrm{ZnTiO}_{3}$ 重复使用催化合成 $\mathrm{H}_{2} \mathrm{O}_{2}$ 性能曲线(网络版 彩图) 


\subsection{3 $\mathrm{H}_{2} \mathrm{O}_{2}$ 在催化剂上的分解}

考察了 $\mathrm{H}_{2} \mathrm{O}_{2}$ 在钛基催化剂上的分解行为. 从 $\mathrm{H}_{2} \mathrm{O}_{2}$ 的分解曲线分析, 如图8所示, $\mathrm{H}_{2} \mathrm{O}_{2}$ 在 $\mathrm{P} 25$ 上分解最快, 在反应 90 min时, $\mathrm{H}_{2} \mathrm{O}_{2}$ 分解了初始浓度的约 $90 \%$, 而在 $\mathrm{ZnTi}-\mathrm{MMO}$ 上分解了 $27 \%$, 在 $\mathrm{TiO}_{2}-\mathrm{ZnTiO}_{3}$ 上分解了 $17 \%$, 而且基本不再随时间继续分解, 表明形成 $\mathrm{TiO}_{2}-\mathrm{ZnTiO}_{3}$ 异质结构可以抑制 $\mathrm{H}_{2} \mathrm{O}_{2}$ 分解. 采用一级反应动力学方程 $\ln \left\{\left[C_{0}\right] /[C]\right\}=K_{t}$ 对分解曲线进行拟合, 得到分解速率常 数 $K . \mathrm{P} 25$ 作催化剂时的 $K=0.01942 \mathrm{~min}^{-1}$, 是ZnTi-MMO 作为催化剂时的 2.17 倍 $\left(K=0.00891 \mathrm{~min}^{-1}\right)$, 是 $\mathrm{TiO}_{2^{-}}$

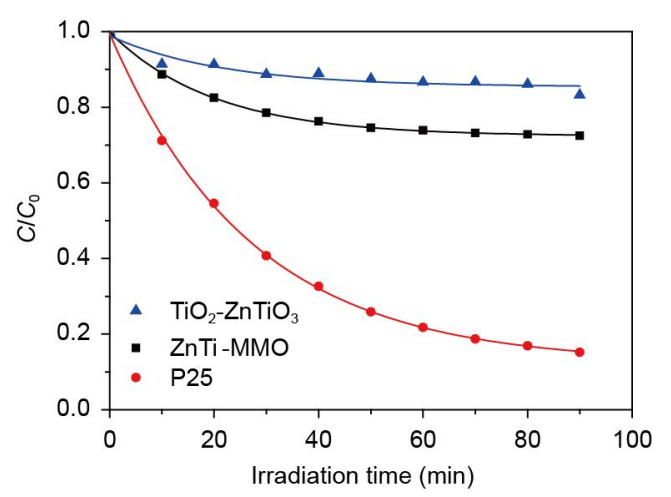

图 $8 \mathrm{H}_{2} \mathrm{O}_{2}$ 在 $\mathrm{TiO}_{2}-\mathrm{ZnTiO}_{3} 、 \mathrm{ZnTi}-\mathrm{MMO} 、 \mathrm{P} 25$ 上的分解曲线 (初始 $\mathrm{H}_{2} \mathrm{O}_{2}$ 浓度 $C_{0}=5 \mathrm{mM}$ ). 反应条件为: $1 \mathrm{~g} / \mathrm{L}$ 的光催化剂, $5 \mathrm{vol} \%$ 的异丙醇, $\mathrm{pH}=3.0$, 氧平衡状态, 氙灯光源, 波长 $>320$ $\mathrm{nm}$, 光照强度为 $100 \mathrm{~mW} / \mathrm{cm}^{2}$ (网络版彩图)

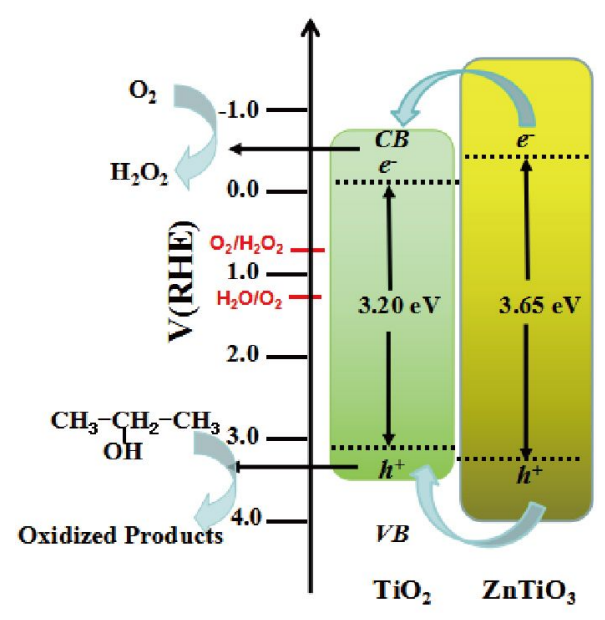

图 $9 \mathrm{TiO}_{2}-\mathrm{ZnTiO}_{3}$ 的能带位置与载流子转移示意图(网络版 彩图)
$\mathrm{ZnTiO}_{3}$ 的 8.59 倍 $\left(K=0.00226 \mathrm{~min}^{-1}\right)$. 这证实了 $\mathrm{H}_{2} \mathrm{O}_{2}$ 在 P25上的强烈分解反应是导致其产量低的重要因素.

\subsection{4 反应机理讨论}

对 $\mathrm{TiO}_{2}-\mathrm{ZnTiO}_{3}$ 的能带结构与光生载流子转移的 可能路径进行了分析, 如图9所示. 锐钣矿型 $\mathrm{TiO}_{2}$ 的导 带和价带位置分别位于 -0.16 和 $+3.04 \mathrm{eV}, \mathrm{ZnTiO}_{3}$ 的导 带和价带位置分别位于 -0.46 和 $+3.19 \mathrm{eV}^{[20,21]}$. $\mathrm{TiO}_{2}$ 的 价带具有比水氧化 $\left(E_{\mathrm{H}_{2} \mathrm{O} / \mathrm{O}_{2}}=+1.23 \mathrm{~V}\right)$ 更正的电势, 光生 空穴有能力氧化水或氧化醇类 ${ }^{[22]}$. $\mathrm{TiO}_{2}$ 的导带具有比 氧还原 $\left(E_{\mathrm{O}_{2} / \mathrm{H}_{2} \mathrm{O}_{2}}=+0.69 \mathrm{~V}\right)$ 更负的电势, 光生电子有能力 还原氧气 ${ }^{[23]}$. 当 $\mathrm{ZnTiO}_{3}$ 与 $\mathrm{TiO}_{2}$ 形成异质结构时, $\mathrm{ZnTiO}_{3}$ 中激发产生的光生电子能够经相界面转移到 $\mathrm{TiO}_{2}$ 的导 带, 促进电子参与的氧还原成 $\mathrm{H}_{2} \mathrm{O}_{2}$; 产生的光生空穴 能够转移到 $\mathrm{TiO}_{2}$ 的价带, 促进空穴参与的水(或醇)氧 化, 形成 $\mathrm{TiO}_{2}-\mathrm{ZnTiO}_{3}$ 异质结构, 既提高了催化剂的氧 还原能力, 又增强了水(或醇)氧化能力. 因此, $\mathrm{H}_{2} \mathrm{O}_{2}$ 在 $\mathrm{TiO}_{2}-\mathrm{ZnTiO}_{3}$ 上具有更大的生成速率. 而如何有效抑制 $\mathrm{H}_{2} \mathrm{O}_{2}$ 在 $\mathrm{TiO}_{2}-\mathrm{ZnTiO}_{3}$ 上分解是进一步提高其催化性能 的关键, 将在以后的工作中详细研究.

\section{4 结论}

以 ZnTi-LDHs 为前驱体, 通过焙烧转化为ZnTi$\mathrm{MMO}$, 作为光驱动合成 $\mathrm{H}_{2} \mathrm{O}_{2}$ 的催化剂, 反应仅以水、 氧气和少量醇为原料即可发生. 在反应过程中, ZnTi$\mathrm{MMO}$ 催化剂中的 $\mathrm{ZnO}$ 逐渐溶蚀, 原位转化为 $\mathrm{TiO}_{2}-$ $\mathrm{ZnTiO}_{3}$ 相. 由原位溶蚀得到的 $\mathrm{TiO}_{2}-\mathrm{ZnTiO}_{3}$ 形成具有 紧密接触界面的异质结构. $\mathrm{H}_{2} \mathrm{O}_{2}$ 在 $\mathrm{TiO}_{2}-\mathrm{ZnTiO}_{3}$ 催化剂 上具有更大的生成速率常数, 而在P 25 上分解更快, 表 明形成 $\mathrm{TiO}_{2}-\mathrm{ZnTiO}_{3}$ 异质结构可抑制 $\mathrm{H}_{2} \mathrm{O}_{2}$ 的分解, 从而 提高 $\mathrm{H}_{2} \mathrm{O}_{2}$ 的生成量. 从光合成 $\mathrm{H}_{2} \mathrm{O}_{2}$ 反应动力学结果分 析, $\mathrm{TiO}_{2}-\mathrm{ZnTiO}_{3}$ 作催化剂时, $\mathrm{H}_{2} \mathrm{O}_{2}$ 生成速率常数大于 ZnTi-MMO和 P25. 从 $\mathrm{H}_{2} \mathrm{O}_{2}$ 在催化剂上的分解动力学 结果分析, $\mathrm{H}_{2} \mathrm{O}_{2}$ 在 $\mathrm{TiO}_{2}-\mathrm{ZnTiO}_{3}$ 上的分解速率常数小于 $\mathrm{ZnTi}-\mathrm{MMO}$ 和P25. 这是 $\mathrm{TiO}_{2}-\mathrm{ZnTiO}_{3}$ 相具有更优催化 性能的主要原因. $\mathrm{TiO}_{2}-\mathrm{ZnTiO}_{3}$ 催化剂具有优异的重复 使用性能, 经过4次重复使用, 催化性能没有明显降低. 本工作加深了对钛基催化剂光合成 $\mathrm{H}_{2} \mathrm{O}_{2}$ 反应动力学 的认识, 对钛基催化剂结构设计有一定的指导. 


\section{参考文献}

1 Hage R, Lienke A. Angew Chem Int Ed, 2006, 45: 206-222

2 Campos-Martin JM, Blanco-Brieva G, Fierro JLG. Angew Chem Int Ed, 2006, 45: 6962-6984

3 Edwards J, Solsona B, Landon P, Carley A, Herzing A, Kiely C, Hutchings G. J Catal, 2005, 236: 69-79

4 Pritchard JC, He Q, Ntainjua EN, Piccinini M, Edwards JK, Herzing AA, Carley AF, Moulijn JA, Kiely CJ, Hutchings GJ. Green Chem, 2010, 12: $915-921$

5 Cai R, Kubota Y, Fujishima A. J Catal, 2003, 219: 214-218

6 Tsukamoto D, Shiro A, Shiraishi Y, Sugano Y, Ichikawa S, Tanaka S, Hirai T. ACS Catal, 2012, 2: 599-603

7 Teranishi M, Naya S, Tada H. J Am Chem Soc, 2010, 132: 7850-7851

8 Maurino V, Minero C, Mariella G, Pelizzetti E. Chem Commun, 2005, 2627

9 He W, Wang R, Zhang L, Zhu J, Xiang X, Li F. J Mater Chem A, 2015, 3: 17977-17982

10 He W, Yang Y, Wang L, Yang J, Xiang X, Yan D, Li F. ChemSusChem, 2015, 8: 1568-1576

11 Yao L, Wei D, Yan D, Hu C. Chem Asian J, 2015, 10: 630-636

12 Tang Y, Wang R, Yang Y, Yan D, Xiang X. ACS Appl Mater Interf, 2016, 8: 19446-19455

13 Wang R, Pan K, Han D, Jiang J, Xiang C, Huang Z, Zhang L, Xiang X. ChemSusChem, 2016, 9: 2470-2479

14 王瑞瑞, 赵有璟, 邵明飞, 项顼, 段雪. 化工学报, 2016, 67: 54-72

5 Li Y, Zhang L, Xiang X, Yan D, Li F. J Mater Chem A, 2014, 2: 13250-13258

Moon G, Kim W, Bokare AD, Sung N, Choi W. Energy Environ Sci, 2014, 7: 4023-4028

Ehsan MA, Khaledi H, Pandikumar A, Rameshkumar P, Huang NM, Arifin Z, Mazhar M. New J Chem, 2015, 39: 7442-7452

Han C, Yang MQ, Weng B, Xu YJ. Phys Chem Chem Phys, 2014, 16: 16891-16903

Xiang X, He W, Xie L, Li F. Catal Sci Technol, 2013, 3: 2819-2827

Reddy KH, Martha S, Parida KM. Inorg Chem, 2013, 52: 6390-6401

Torimoto T, Nakamura N, Ikeda S, Ohtani B. Phys Chem Chem Phys, 2002, 4: 5910-5914

Cai Y, Ye Y, Tian Z, Liu J, Liu Y, Liang C. Phys Chem Chem Phys, 2013, 15: 20203-20209

23 Mrowetz M, Selli E. J Photochem Photobiol A Chem, 2006, 180: 15-22 


\title{
In-situ conversion and catalytic properties of mixed-metal oxide catalysts for photosynthesis of hydrogen peroxide
}

\author{
Dandan Han, Jianyi Liu, Junjiao Yang, Xu Xiang* \\ State Key Laboratory of Chemical Resource Engineering, Beijing University of Chemical Technology, Beijing 100029, China \\ *Corresponding author (email: xiangxu@mail.buct.edu.cn)
}

\begin{abstract}
Titanium-based mixed-metal oxide (MMO), acting as a catalyst for light-driven generation of hydrogen peroxide $\left(\mathrm{H}_{2} \mathrm{O}_{2}\right)$, was obtained by calcining a titanium-based layered double hydroxide precursor. The findings indicated that the $\mathrm{ZnO}$ phase within the MMO was etched and the MMO was transformed into $\mathrm{TiO}_{2}-\mathrm{ZnTiO}_{3}$ heterostructure during the synthesis of $\mathrm{H}_{2} \mathrm{O}_{2}$. Both the $\mathrm{MMO}$ and $\mathrm{TiO}_{2}-\mathrm{ZnTiO}_{3}$ are superior to the commercial $\mathrm{TiO}_{2}(\mathrm{P} 25: 80 \%$ anatase and $20 \%$ rutile) for photosynthesis of $\mathrm{H}_{2} \mathrm{O}_{2}$. The generation of $\mathrm{H}_{2} \mathrm{O}_{2}$ was determined by both the formation rate and the decomposition rate. The kinetic studies revealed that the formation rate constant $\left(K_{\mathrm{f}}\right)$ is $3.581 \mu \mathrm{M} / \mathrm{min}$ on the $\mathrm{TiO}_{2}-\mathrm{ZnTiO}_{3}, 3.65$ times larger than that on the MMO $\left(K_{\mathrm{f}}=0.982 \mu \mathrm{M} / \mathrm{min}\right)$ and also 4.2 times larger than that on P25 $\left(K_{\mathrm{f}}=0.852 \mu \mathrm{M} / \mathrm{min}\right)$. And the decomposition rate constant $\left(K_{\mathrm{d}}\right)$ of $\mathrm{H}_{2} \mathrm{O}_{2}$ on the $\mathrm{TiO}_{2}-\mathrm{ZnTiO}_{3}$ is $0.0472 \mathrm{~min}^{-1}, 3.97$ times larger than that on the MMO $\left(K_{\mathrm{d}}=0.0119 \mathrm{~min}^{-1}\right)$ and 1.26 times larger than that on P25 $\left(K_{\mathrm{d}}=0.0374 \mathrm{~min}^{-1}\right)$. The in situ generated $\mathrm{TiO}_{2}-\mathrm{ZnTiO}_{3}$ possessed larger $K_{\mathrm{f}}$ and $K_{\mathrm{d}}$ values. Consequently, the yield of $\mathrm{H}_{2} \mathrm{O}_{2}$ reached the same level on the $\mathrm{TiO}_{2}-\mathrm{ZnTiO}_{3}$ and the MMO with a longer reaction period, although the former catalyzed a higher yield of $\mathrm{H}_{2} \mathrm{O}_{2}$ at the beginning of the reaction. This result hinted that the reaction kinetics was affected by the phase change of the catalyst whereas the final yield of $\mathrm{H}_{2} \mathrm{O}_{2}$ was hardly impacted. The key way to enhance the yield of $\mathrm{H}_{2} \mathrm{O}_{2}$ could be the inhibition of $\mathrm{H}_{2} \mathrm{O}_{2}$ decomposition on the surface of catalyst. This work intensified the understanding of reaction kinetics regarding the photosynthesis of $\mathrm{H}_{2} \mathrm{O}_{2}$ on the Ti-based MMO and directed the structural design of the oxide photocatalyst to some extent.
\end{abstract}

Keywords: mixed-metal oxide, hydrogen peroxide, photosynthesis, reaction kinetics

doi: $10.1360 / \mathrm{N} 032016-00232$ 